\title{
Class I hysterectomy in stage la2-Ib1 cervical cancer
}

\author{
Long Chen, Wei-Na Zhang, Sheng-Miao Zhang, Yuan Gao, Tian-Hong Zhang, Ping Zhang \\ Department of Gynecology, Qingdao Municipal Hospital, Qingdao, China
}

Videosurgery Miniinv 2018; 13 (4): 494-500

DOI: https://doi.org/10.5114/wiitm.2018.76832

\begin{abstract}
Introduction: During the last 3 decades, the standard treatment for stage la2-Ib1 cervical cancer has been Piver-Rutledge class II or III radical hysterectomy. However, this surgery is associated with a high rate of urologic morbidity. Aim: To determine the efficacy of class I radical hysterectomy compared with class III radical hysterectomy in terms of morbidity, overall survival, and patterns of relapse in patients with la2-lb1 cervical cancer undergoing primary surgery. Material and methods: A total of 101 patients with stage la2-lb1 cervical cancer $<2 \mathrm{~cm}$ were randomized to class I and class III hysterectomy groups. Clinical, pathologic, and follow-up data were prospectively collected. Univariate analysis was carried out. Of the total patients, 45 were randomized to class I surgery and 56 to class III surgery. No significant differences were observed in terms of pathologic findings or adjuvant treatment $(p>0.05)$. The morbidity rates were higher after class III surgery.

Results: The difference in recurrence rate between the class I and class III groups was not statistically significant $(p>0.05)$. The 5-year overall survival rate was $93 \%$ and $91 \%$, respectively $(p>0.05)$. There were no significant differences in terms of recurrence rate or overall survival among patients with stage la2-Ib1 cervical cancer $2 \mathrm{~cm}$ who underwent class I or radical (class III) hysterectomy. Morbidity was proportional to the extent of radicality.

Conclusions: These data confirm the need for reducing surgical radicality in the treatment of patients with early cervical cancer, by tailoring the extent of resection according to the extent of disease.
\end{abstract}

Key words: cervical cancer, class I hysterectomy, radical hysterectomy

\section{Introduction}

Cervical cancer remains the most common gynecologic malignancy worldwide [1]. To date, the standard treatment for early-stage cervical cancer (la2-lb1) is radical hysterectomy with pelvic lymphadenectomy [2]. However, it causes many complications such as early urinary tract infection, bladder atony, lymphedema, ileus, vesicovaginal fistula, and sexual dysfunction [3]. Therefore, it is important to identify patients with early invasive cancer whose lesions could be resected using a less radical surgery.

In patients with a diagnosis of early cervical cancer, the possibility of infiltration of parametrial tissue is small and the risk of lymph node metas- tasis is very low. Therefore, for these patients, it is important to determine whether there is a more suitable operation that could minimize the operative complications without affecting the prognosis. Many researchers have investigated this issue. Van Meurs et al. reported that there was no infiltration of parametrial tissue in 1063 patients with stage la2 cervical cancer, but lymph node metastasis was present in $4.8 \%$ of the patients [4]. Covens reported that among 842 patients with stage Ib1 cervical cancer, only $0.6 \%$ of low-risk patients (tumor diameter $<2 \mathrm{~cm}$, stromal invasion < $10 \mathrm{~mm}$, no pelvic lymph node metastasis) had a risk of parametrial tissue infiltration [5]. Kinney et al. [6] reported that none of the 83 patients with

\section{Address for correspondence}

Ping Zhang, Department of Gynecology, Qingdao Municipal Hospital, 1 Jiaozhou Road, 266000 Qingdao, China, phone: +86 18661678290 , e-mail: pingzhangdoc@163.com 
stage Ib cervical cancer with a tumor diameter of $\leq 2 \mathrm{~cm}$ had parametrial lymph node metastasis. Thus, they questioned the necessity of parametrial tissue resection in patients with early-stage cervical cancer. Schmeler et al. reported that approximately $60 \%$ of patients who underwent a radical trachelectomy had no residual disease in their surgical specimen, which perhaps indicates that those patients could have been treated with less radical surgery [7]. The results of these studies suggest that the surgical removal of the parametrium may not be necessary in patients with stage lb1 cervical cancer with tumors $\leq 2 \mathrm{~cm}$. In these patients, less radical surgery in conjunction with pelvic lymphadenectomy may be worth considering.

The principle of surgical treatment for cervical cancer is to remove the lesion, minimize complications, and improve the quality of life [8]. The objective of this study was to determine whether class I hysterectomy and bilateral pelvic lymphadenectomy can be suitable for patients with early-stage cervical cancer with tumor size $<2 \mathrm{~cm}$ and negative lymph node metastasis, in order to minimize the damage caused by surgery.

\section{Aim}

Moreover, we aimed to determine the efficacy of class I simple hysterectomy compared with class III radical hysterectomy (complete extirpation of the parametria) in terms of morbidity, overall survival, disease-free survival, and recurrence rates.

\section{Material and methods}

After obtaining institutional review board approval, a prospective randomized study was carried out from January 2006 to January 2011 in the Department of Obstetrics and Gynecology of Qingdao Municipal Hospital.

\section{Patients}

We collected data on all patients with stage la2Ib1 cervical cancer with tumor diameter $<2 \mathrm{~cm}$. The initial histological examination before surgery was performed on biopsy specimens. The stage was confirmed by at least 2 experienced gynecology professors. Chest radiography and intravenous pyelography evaluations were also conducted. No lymph node metastasis was found on positron emission computed tomography or magnetic resonance imaging per- formed before the surgery. When the clinical tumor size was $>2 \mathrm{~cm}$, neoadjuvant chemotherapy (NACT) was administered before surgery. Cisplatin-based adjuvant chemotherapy was administered in 1-2 courses before surgery depending on the patient's tolerance and response. If the tumor size was $<2 \mathrm{~cm}$ after NACT, the patients were considered eligible for inclusion. This study was conducted in accordance with the Declaration of Helsinki. This study was conducted with approval from the Ethics Committee of Qingdao Municipal Hospital. Written informed consent was obtained from all participants.

\section{Treatment}

Written consent was obtained from all patients before randomization. Eligible patients were randomized to receive either Piver-Rutledge class I or class III radical hysterectomy. The treatment was assigned using block randomization (clusters of 10 cases) from a computer-generated table created before starting the trial; the treatment assignments were unknown to the investigators and were decoded after obtaining informed consent from the patient.

All operations were performed by board-certified gynecological oncology surgeons who were skilled at laparoscopic operations at the Qingdao Municipal Hospital. All procedures were performed laparoscopically. Class I procedures included extrafascial hysterectomy, bilateral salpingo-oophorectomy, and removal of the upper third of the vagina. Laparoscopic class III procedures comprised the removal of all anatomical structures mentioned in the Introduction. Both surgical treatments included bilateral pelvic lymphadenectomy and were performed according to Piver-Rutledge-Smith classification 1 by the same surgical team. Pelvic lymphadenectomy was done, and no lymph node metastasis was found in quick paraffin sections. Patients with lymph node metastasis were considered ineligible for this study.

Paracervical involvement, positive or close $(<3 \mathrm{~mm})$ resection margins, lymph-vascular space invasion close to the resection margins, and poorly differentiated tumor were considered risk factors requiring adjuvant chemotherapy or radiotherapy.

Follow-up consisted of a pelvic examination every 3 months during the first 2 years, twice a year from the $3^{\text {rd }}$ to the $5^{\text {th }}$ year, and then yearly thereafter. Intravenous pyelography was performed in all patients 30 days after the operation. Ultrasound scan of the pelvis and 
Pap smear test were scheduled every 6 months. Furthermore, chest radiographs were taken yearly.

The patients remained catheterized from the operation until the residual urine volume was $<100 \mathrm{ml}$. All women who underwent class I or class III surgery were asked to complete a questionnaire about their symptoms of frequency/urgency, nocturia, dysuria, urinary retention, incontinence, bowel rectal function, fecal incontinence, and air incontinence, and their ability to distinguish air from feces 1 year after the surgery.

\section{Statistical analysis}

The $\chi^{2}$ test with Yates' continuity correction was used to test the association between 2 variables. Quantitative data were compared using 2-way analysis of variance. Univariate analysis was carried out with the survival curves generated using the Kaplan-Meier method and compared with the log-rank test.

\section{Results}

\section{Baseline characteristics and treatment analysis}

Among all patients with stage la2-lb1 cervical cancer treated with upfront surgery at our institute

Table I. Patients' baseline characteristics and pathologic findings

\begin{tabular}{|c|c|c|c|}
\hline Parameter & Class I & Class III & $P$-value \\
\hline \multicolumn{4}{|l|}{ FIGO stage: } \\
\hline la2 & 25 & 30 & \\
\hline Ib1 & 20 & 26 & 0.07 \\
\hline \multicolumn{4}{|l|}{ Age [years]: } \\
\hline 40 & 6 & 10 & \\
\hline $41-50$ & 20 & 22 & \\
\hline $51-60$ & 16 & 22 & \\
\hline$>60$ & 3 & 2 & \\
\hline Median (range) & $50(34-75)$ & $47(24-72)$ & \\
\hline \multicolumn{4}{|l|}{ Pathologic findings: } \\
\hline Adenocarcinoma & 10 & 7 & 0.4 \\
\hline Grade 3 & 14 & 17 & 0.5 \\
\hline LVSI & 10 & 15 & 0.9 \\
\hline Parametrial invasion & 0 & 2 & \\
\hline \multicolumn{4}{|l|}{ Corpus uteri invasion: } \\
\hline Micrometastasis & 4 & 6 & \\
\hline Macrometastasis & 9 & 5 & \\
\hline
\end{tabular}

during the study period, 101 were eligible for inclusion. A total of 45 patients were randomized to class I radical hysterectomy and 56 patients to class III radical hysterectomy. The patients' characteristics are summarized in Table I. The pathologic findings are also reported in Table I. No statistically significant differences among all characteristics examined using the pathologic specimens were observed between the 2 groups. A total of 45 (44\%) patients received adjuvant therapy for the following reasons: vascular space invasion, involvement of margins, and grade 3 tumor. Chemotherapy and radiotherapy were administered to 22 patients in the class I group and 23 patients in the class III group.

\section{Follow-up}

The minimum follow-up time was 60 months. We observed $5(11 \%)$ recurrences in patients in the class I group, all of whom had received radiation therapy. We also observed 10 (18\%) recurrences in patients in the class III group, of whom 7 had received radiation therapy and 3 had not. The differences observed were not statistically significant $(p>0.05)$ (Figure 1). Of the patients with relapse, $89 \%$ in the class I group and $82 \%$ in the class III group are currently alive with no evidence of disease after salvage therapy.

The overall 5-year survival was 93\% (42 of 45) for patients who underwent class I surgery and $91 \%$



Figure 1. Overall survival curves of two groups by Kaplan-Meier method $(p=0.339)$ 
(51 of 56) for those who underwent class III surgery $(p>0.05)$. This difference is attributable to 8 cases, as 42 patients are currently alive in the class I group versus 51 patients in the class III arm.

Univariate analysis for overall survival (Table II) showed that tumor grade was the most predictive risk factor $(p<0.05)$. Other interesting parameters, although with non-significant $p$-values, were histotype $(p=0.2)$ and left ventricular systolic index $(p=0.3)$.

Morbidity was higher in the class III group than in the class I group. The mean duration of postoperative catheterization in the class I group was 7.03 days, which was much shorter than that in the class II group (15.13 days, $p<0.001)$. Urinary symptoms were evaluated 1 year after the surgery. There was a significant reduction in the incidence of postoperative self-catheterization and a reduction in the period of bladder function recovery in patients in the class I group. At 1 year postoperatively, urinary complications such as nocturia, urinary urgency and frequency, postoperative urine retention, and dysuria were significantly different (Table III). The time of passage of gas through the anus was shorter (30.97 $\pm 1.80 \mathrm{~h}$ ) in patients in the class I group than in those in the class III group $(55.55 \pm 3.01 \mathrm{~h})$. The intestinal function of patients in the class I group recovered better than that of patients in the class III group. In the analysis of the questionnaire responses, no significant differences were detected between the 2 groups in terms of bowel-rectal quality of life. The rates of surgery-related complications were higher in the class III group, including lymphocyst and leg edema. However, there was no significant difference in deep venous thrombosis and pulmonary embolism.

\section{Discussion}

Our study is a prospective randomized trial comparing class I and class III radical hysterectomy in patients with early-stage cervical cancer with tumor size $<2 \mathrm{~cm}$ and negative lymph node metastasis. We observed that the morbidity was higher in the class III group than in the class I group, and that there was no significant difference in overall 5-year survival between the 2 groups.

Positive lymph node involvement is classified as a high-risk factor in cervical cancer [9]. In our study, we excluded patients with positive lymph node metastasis detected on biopsy specimens from surgery. The most recent literature reports show that paracervical invasion is nearly absent in selected tumors

Table II. Univariate analysis for overall survival

\begin{tabular}{|c|c|c|c|c|}
\hline Variables & Level & $N$ & Patients with NED & $P$-value \\
\hline \multirow[t]{2}{*}{ Type of surgery } & Class I & 45 & $44(71 \%)$ & 0.1 \\
\hline & Class III & 56 & $51(81 \%)$ & \\
\hline \multirow[t]{2}{*}{ Age [years] } & $\leq 50$ & 80 & $64(80 \%)$ & 0.9 \\
\hline & $>50$ & 21 & $15(72 \%)$ & \\
\hline \multirow[t]{2}{*}{ FIGO stage } & la2 & 50 & $38(77 \%)$ & 0.87 \\
\hline & lb1 & 51 & $34(67 \%)$ & \\
\hline \multirow[t]{2}{*}{ Histotype } & Squamous & 84 & $67(80 \%)$ & 0.2 \\
\hline & Adenocarcinoma & 17 & $9(53 \%)$ & \\
\hline \multirow[t]{3}{*}{ Grade } & G1 & 31 & 25 (81\%) & \\
\hline & G2 & 44 & $31(71 \%)$ & \\
\hline & G3 & 26 & $12(48 \%)$ & \\
\hline \multirow[t]{2}{*}{ Parametria } & Negative & 99 & $59(60 \%)$ & 0.52 \\
\hline & Positive & 2 & $1(50 \%)$ & \\
\hline \multirow[t]{2}{*}{ Corpus uteri } & Negative & 86 & $63(74 \%)$ & 0.26 \\
\hline & Positive & 15 & $12(85 \%)$ & \\
\hline \multirow[t]{2}{*}{ LVSI } & Absent & 84 & $66(79 \%)$ & 0.3 \\
\hline & Present & 17 & $12(71 \%)$ & \\
\hline
\end{tabular}

NED - no evidence of disease, LVSI - lymph-vascular space invasion. 
Table III. Treatment-related morbidity in patients undergoing class I and class III hysterectomy

\begin{tabular}{|c|c|c|c|}
\hline Characteristics & Class I $(n=45)$ & Class III $(n=56)$ & $P$-value \\
\hline \multicolumn{4}{|l|}{ DPC [days]: } \\
\hline Mean \pm SD & $7.03 \pm 2.21$ & $15.13 \pm 3.00$ & $<0.001$ \\
\hline Frequency/urgency symptoms & 3 & 15 & $<0.01$ \\
\hline Nocturia & 2 & 13 & $<0.01$ \\
\hline Dysuria & 1 & 9 & $<0.05$ \\
\hline Urinary retention & 0 & 7 & $<0.05$ \\
\hline Incontinence & 5 & 17 & $<0.01$ \\
\hline \multicolumn{4}{|l|}{ Passage of gas by anus: } \\
\hline Hours after surgery & $30.97 \pm 1.80$ & $55.50 \pm 3.01$ & $<0.001$ \\
\hline \multicolumn{4}{|l|}{ Bowel rectal function: } \\
\hline Normal & $28(93 \%)$ & $32(91 \%)$ & \\
\hline Constipation & $2(7 \%)$ & $3(9 \%)$ & \\
\hline \multicolumn{4}{|l|}{ Fecal incontinence: } \\
\hline Never & 0 & 0 & NA \\
\hline Seldom & 0 & 4 & \\
\hline \multicolumn{4}{|l|}{ Air incontinence: } \\
\hline Never & 0 & 0 & NA \\
\hline Seldom & 0 & 2 & \\
\hline \multicolumn{4}{|l|}{ Able to distinguish air from feces: } \\
\hline No & 0 & 0 & NA \\
\hline Not always & 0 & 3 & \\
\hline Lymphocyst & 3 & 12 & $<0.05$ \\
\hline Leg edema & 2 & 10 & $<0.05$ \\
\hline Deep venous thrombosis & 2 & 3 & $>0.05$ \\
\hline Pulmonary embolism & 0 & 0 & \\
\hline
\end{tabular}

$D P C$ - mean duration of postoperative catheterization.

[10-14]. The selection criteria differ among studies; however, overall, small tumors (i.e., $<2 \mathrm{~cm}$ ) with negative lymph nodes showed few or no signs of parametrial spread, and therefore should not require the removal of the entire parametria to be effectively cured.

With the development of technology, there emerged a new, effective method to identify sentinel lymph node metastasis. Sentinel lymph node mapping by lymphoscintigraphy, intraoperative intracervical blue dye injection, or indocyanine green with near infrared fluorescence mapping could be used to identify sentinel lymph node metastasis $[15,16]$. We could use sentinel lymph node mapping in the next study to exclude lymph node metastasis.

The use of modified radical hysterectomy may be not only curative but also minimally invasive for patients with stage la2-Ib1 cancer with a tumor $<2 \mathrm{~cm}$ in diameter [17]. On the basis of these studies, we strictly selected patients with cervical cancer with tumor size $<2 \mathrm{~cm}$ and negative lymph node metastasis to investigate whether modified radical hysterectomy could be effective without affecting the prognosis of these patients. We attempted to investigate the necessity of tailoring the procedure according to the extent of the disease, in order to decrease the overall morbidity caused by the surgical treatment. Complications represent a difficult problem, and the type of surgery greatly influences the morbidity rate [18]. We observed fewer surgery-related complications such as lymphocyst and leg edema in the class I group. The quality of life of patients in the class I group evaluated 1 year after the oper- 
ation was obviously better than that of patients in the class III group. This confirms that the function of organs within the operative field was preserved and surgery-related symptoms that degrade the quality of life, including dysuria, constipation, and sexual dissatisfaction, were relieved. Thus, patients who underwent class I hysterectomy were less disturbed by the adverse effects of surgery, which improved their emotional stability and enabled them to return to work or normal activities.

We found no significant difference in overall 5 -year survival between the 2 groups. Other similar studies have been reported in previous years. In 1987, Stark reported 208 cases of stage IB cervical carcinoma treated with either class III radical hysterectomy or class I hysterectomy, with the class I procedure preserving a large part of the parametrial tissue. The survival rate was lower and the morbidity was higher in the class III group than in the Galvine-TeLinde group (72.3\% vs. 78.5\%) [19]. This observation was also supported by the studies of Landoni et al. and Sartori et al. [20, 21], which suggested that radical removal of the lateral and posterior parametrial tissue cannot be performed on the anterior portion, because $92 \%$ of cases had $\leq 3 \mathrm{~mm}$ intact cervical stroma; therefore, appropriate radicality is hardly ever achieved and is independent from the lateral-posterior removal of paracervical tissue.

Tumor markers of cervical cancer may be useful not only for early diagnosis but also in others fields of application such as evaluation and monitoring of treatments. Valenti et al. reported that CEA, SCC-Ag, and CD44 could be used to evaluate the potential for local and peripheral spread, the progression tendency and the aggressiveness of advanced CC [22]. We could compare the tumor marker changes in the two groups in the next step.

The established concept of maximum surgical radicality to achieve the maximum chance of cure should be revised. Although surgery is effective and useful for the curative treatment of patients with early invasive cervical cancer, minimization of the extent of surgical procedures provides many additional advantages for patients. Because the mean age of patients at the time of diagnosis of cervical precancer and invasive cancer has been decreasing, the need for minimization of surgery to improve the quality of life is increasing. As already stated, new minimally invasive approaches to perform nerve-sparing techniques have reduced the morbidity rates [23]; nevertheless, significant complication rates in class III radical hysterectomies were still observed in the most recent studies.

\section{Conclusions}

Our results suggest that there are no differences in terms of pattern of recurrence and survival in patients with stage la2-lb1 cervical tumors with a tumor diameter of $<2 \mathrm{~cm}$ treated with class I or class III hysterectomy; however, the same conclusion does not apply to cases of larger tumors.

Nevertheless, our promising results should be confirmed by future studies. The present trial only serves as an encouragement to other surgeons to apply our selection criteria in attempting to reduce surgical radicality in the treatment of early cervical cancer.

\section{Acknowledgments}

Long Chen and Wei-Na Zhang are co-first authors. Financial sources - Qingdao Outstanding Health Professional Development Fund.

\section{Conflict of interest}

The authors declare no conflict of interest.

\section{References}

1. Jemal A, Bray F, Center MM, et al. Global cancer statistics. CA Cancer J Clin 2011; 61: 69-90.

2. Ramirez PT, Pareja R, Rendón GJ, et al. Management of lowrisk early-stage cervical cancer: should conization, simple trachelectomy, or simple hysterectomy replace radical surgery as the new standard of care? Gynecol Oncol 2014; 132: 254-9.

3. Hacker NF, Friedlander ML. Cervical cancer. In: Gynecologic Oncology. $5^{\text {th }}$ ed. Berek JS, Hacker NF (eds). Wolters Kluwer, Philadelphia 2010; 341-95

4. van Meurs $\mathrm{H}$, Visser $\mathrm{O}$, Buist MR, et al. Frequency of pelvic lymph node metastases and parametrial involvement in stage IA2 cervical cancer: a population-based study and literature review. Int J Gynecol Cancer 2009; 19: 21-6.

5. Covens A, Rosen B, Murphy J, et al. How important is removal of the parametrium at surgery for carcinoma of the cervix? Gynecol Oncol 2002; 84: 145-9.

6. Kinney WK, Hodge DO, Egorshin EV, et al. Identification of a lowrisk subset of patients with stage IB invasive squamous cancer of the cervix possibly suited to less radical surgical treatment. Gynecol Oncol 1995; 57: 3-6.

7. Schmeler KM, Frumovitz M, Ramirez PT. Conservative management of early stage cervical cancer: is there a role for less radical surgery? Gynecol Oncol 2011; 120: 321-5. 
8. Frumovitz M, Sun CC, Schover LR, et al. Quality of life and sexual functioning in cervical cancer survivors. J Clini Oncol 2005; 23: 7428-36.

9. Matsuo K, Mabuchi S, Okazawa M, et al. Utility of risk-weighted surgical-pathological factors in early-stage cervical cancer. $\mathrm{Br}$ J Cancer 2013; 108: 1348-57.

10. Landoni F, Maneo A, Cormio G, et al. Class II versus class III in stage IB-IIA cervical cancer: a prospective randomized study. Gynecol Oncol 2001; 80: 3-12.

11. Steed H, Capstick V, Schepansky A, et al. Early cervical cancer and parametrial involvement: is it significant? Gynecol Oncol 2006; 103: 53-7.

12. Wright JD, Grigsby PW, Brooks R, et al. Utility of parametrectomy for early stage cervical cancer treated with radical hysterectomy. Cancer 2007; 110: 1281-6.

13. Strnad P, Robova H, Skapa P, et al. A prospective study of sentinel lymph node status and parametrial involvement in patients with small tumour volume cervical cancer. Gynecol Oncol 2008; 109: 280-4.

14. Frumovitz M, Sun CC, Schmeler KM, et al. Parametrial involvement in radical hysterectomy specimens for women with early-stage cervical cancer. Obstet Gynecol 2009; 114: 93-9.

15. Diaz JP, Gemignani ML, Pandit-Taskar N, et al. Sentinel lymph node biopsy in the management of early-stage cervical carcinoma. Gynecol Oncol 2011; 120: 347-52.

16. Rossetti D, Vitale SG, Tropea A, et al. New procedures for the identification of sentinel lymph node: shaping the horizon of future management in early stage uterine cervical cancer. Updates Surg 2017; 69: 383-8.

17. Arimoto T, Kawana K, Adachi K, et al. Minimization of curative surgery for treatment of early cervical cancer: a review. Jpn J Clin Oncol 2015; 45: 611-6.

18. Landoni F, Maneo A, Zapardiel I, et al. Class I versus class III radical hysterectomy in stage IB1-IIA cervical cancer. A prospective randomized study. Eur J Surg Oncol 2012; 38: 203-9.

19. Stark G. Surgical therapy of stage IB cervix cancer. Geburtshilfe Frauenheilkd 1987; 47: 45-8.

20. Landoni F, Bocciolone L, Perego P, et al. Cancer of the cervix, FIGO stages IB and IIA: patterns of local growth and paracervical extension. Int J Gynecol Cancer 1995; 5: 329-34.

21. Sartori E, Fallo L, La Face B, et al. Extended radical hysterectomy in early-stage carcinoma of the uterine cervix: tailoring the radicality. Int J Gynecol Cancer 1995; 5: 143-7.

22. Valenti G, Vitale SG, Tropea A, et al. Tumor markers of uterine cervical cancer: a new scenario to guide surgical practice? Updates Surg 2017; 69: 441-9.

23. Chen L, Zhang WN, Zhang SM, et al. Effect of laparoscopic nerve-sparing radical hysterectomy on bladder function, intes tinal function recovery and quality of sexual life in patients with cervical carcinoma. Asian Pac J Cancer Prev 2014; 15: 10971-5.

Received: 26.03.2018, accepted: 13.05.2018. 